CASE REPORT

\author{
G.-F. Yang \\ S.-Y. Wu \\ L.-J. Zhang \\ G.-M. Lu \\ W. Tian \\ K. Shah
}

\section{Imaging Findings of Extraventricular Neurocytoma: Report of 3 Cases and Review of the Literature}

\begin{abstract}
SUMmARY: Extraventricular neurocytoma (EVN) is rare entity with similar biologic behavior and histopathologic characteristics to neurocytomas that occur in the lateral ventricles, according to the 2007 World Health Organization classification. We report the cases of 3 patients with extraventricular neurocytoma, which presented as large tumors in the left frontal and parietal lobes and in the sellar region. MR imaging showed cystic degeneration, calcification and/or hemorrhage, intense enhancement, and perilesional edema. EVN should be considered in the differential diagnosis for large, heterogeneous, enhancing brain tumors that occur in young people.
\end{abstract}

$\mathbf{E}$ xtraventricular neurocytoma $(\mathrm{EVN})$ was included in the 2000 World Health Organization (WHO) classification of tumors of the central nervous system but remained unclassified until the 2007 revision. This fourth edition of the WHO classification proposed an International Classification of Diseases-O code identical to that of central neurocytoma (9506/ 1). ${ }^{1}$ EVNs show wide variability with regard to morphologic features, cellularity, and proliferation rate and are more frequently associated with poorer outcome than central neurocytoma. ${ }^{2}$ EVNs have been reported in a variety of locations outside of the supratentorial ventricular system but seem to arise most frequently within the cerebral hemispheres. We found few reports of the imaging findings of EVNs. ${ }^{3-5}$ Herein, we report the cases of 3 patients with EVNs, including MR imaging features and histopathologic appearance. These may provide some insight into imaging diagnosis and differential diagnosis of this entity.

\section{Case Reports}

\section{Case 1}

A 24-year-old woman was admitted for headache and 4 days of right hemiparesis. She underwent nonenhanced CT scan, which showed a heterogeneous round mass with multiple calcifications in the left parietal lobe. Extensive perilesional edema, compression of the left lateral ventricle, and midline shift to the right were seen. Later, MR imaging showed a cystic and solid, lobulated mass measuring 4.6 $\mathrm{cm} \times 5.1 \mathrm{~cm} \times 5.3 \mathrm{~cm}$. A fluid-level was present medially. Contrastenhanced MR imaging showed intense enhancement in the lateral component (Fig $1 A, 1 B$, and $1 C$ ). A high-grade neuroepithelial tumor was suspected. Intraoperative findings demonstrated a grayish mass measuring $5 \mathrm{~cm} \times 5 \mathrm{~cm} \times 6 \mathrm{~cm}$. Intratumoral hemorrhage was observed. Hematoxylin-eosin (H\&E) staining showed that the tumor was composed of uniform round cells with a branching capillary web.

Received June 5, 2008; accepted after revision July 17.

From the Departments of Nuclear Medicine (G.-F.Y.) and Medical Imaging (L.-J.Z., G.-M.L.), Jinling Hospital, Clinical School of Medical College, Nanjing University, Nanjing, Jiangsu, China; Medical Imaging Institute of Tianjin (S.-Y.W.), Tianjin, China; Center of Medical Imaging (W.T.), First Affiliated Hospital of Kunming Medical College, Kunming, Yunnan, China; and Department of Diagnostic Radiology (K.S.), University of Texas M.D. Anderson Cancer Center, Houston, Texas.

Please address correspondence to Long-Jiang Zhang, Department of Medical Imaging, Jinling Hospital, Clinical School of Medical College, Nanjing University, 305 Zhongshan East Rd, Xuanwu District, Nanjing, Jiangsu 210002, China

DOI 10.3174/ajnr.A1279
Immunohistochemical staining showed glial fibrillary acidic protein $($ GFAP) $(-)$, vimentin $(\operatorname{Vim})(-)$, S-100 $(+++)$, and synaptophysin (Syn) $(+++)$ (Fig $1 D$ and $1 E)$. The pathologic diagnosis was neurocytoma in the left parietal lobe.

\section{Case 2}

A 2-year-old boy was admitted for moderate right-sided hemiparesis. A CT scan at a local hospital showed an irregular mass in the left frontoparietal lobes with multiple dotted and linear calcifications. MR imaging performed soon after demonstrated a heterogeneously enhancing mass measuring $5.2 \mathrm{~cm} \times 4.1 \mathrm{~cm} \times 3.8 \mathrm{~cm}$ (Fig $2 A,-B)$. Marked edema surrounded the mass. The preoperative diagnosis was oligodendroglioma. At resection, a well-defined $5-\mathrm{cm} \times 6-\mathrm{cm} \times$ 6-cm mass was demonstrated, with marked blood supply and 2 veins around the mass. On sectioning, focal calcification was displayed. $\mathrm{H} \& \mathrm{E}$ staining showed that the tumor was composed of uniform, round cells with a branching capillary web (Fig 2D). Immunohistochemical staining showed neuron-specific enolase (NSE) $(+)$, GFAP $( \pm)$, neurofilament protein $(\mathrm{NF})(+)$, and Syn $(+++)$. Histopathologic examination revealed neurocytoma of the left frontoparietal lobes.

\section{Case 3}

A 46-year-old woman had visual impairment for 1 year that progressed to complete left visual loss 1 month before presentation. CT scan performed in the local hospital displayed an isoattenuating mass in the sellar and suprasellar region with intratumoral calcification. MR examination showed an enhancing, extra-axial mass measuring $3.5 \mathrm{~cm} \times 4.2 \mathrm{~cm}$ in the sellar region. Patchy cystic changes and dotted calcifications were noted (Fig $3 A,-B$ ). Diffusion-weighted images showed high signal intensity within the mass (Fig $3 C$ ). The mass displaced or partially encased adjacent arteries. The preoperative diagnosis was sellar meningioma. The intraoperative finding was an irregular sellar mass that was adherent to the bilateral internal carotid arteries, proximal middle cerebral arteries, and posterior communicating arteries. The left optic nerve was compressed and displaced anteriorly. The superior surface of the mass could not be seen. To avoid damage to adjacent normal tissues, we performed an incomplete resection. Immunohistochemical staining showed NSE $(+)$, GFAP $(-)$, Vim $(++)$, and Syn $(++)$ (Fig $3 E,-F)$. The histopathologic diagnosis was neurocytoma in the sellar region. Postoperative CT scanning showed a $3.2-\mathrm{cm} \times 3.0-\mathrm{cm}$ residual neurocytoma (Fig $3 G)$. 

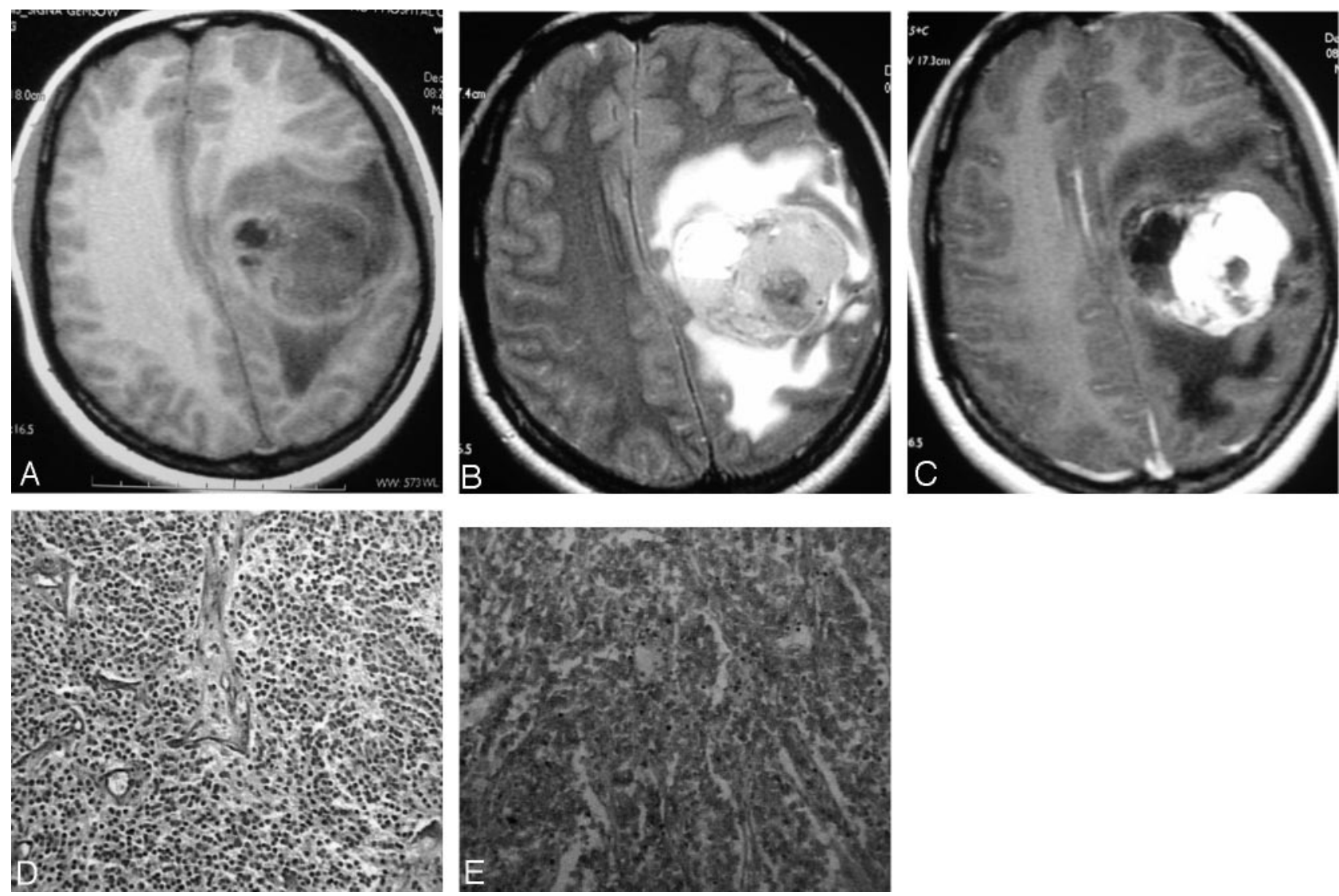

Fig 1. Neurocytoma in the parietal lobe in a 24-year-old woman. $A$, T1-weighted image. $B$, T2-weighted image showing a heterogeneous left parietal mass with a fluid level medially Low signal intensity in the mass was noted on T2-weighted image. $C$, Contrast-enhanced T1-weighted image showing extensive heterogeneous enhancement in the solid part of the mass $D, H \& E$ staining showing that the mass was composed of uniform small round cells. E, Diffuse positive Syn was demonstrated.

\section{Discussion}

Central neurocytomas are rare brain tumors occurring in young adults and usually located in the cerebral ventricles. These neoplasms account for $0.25 \%$ to $0.5 \%$ of all intracranial tumors. ${ }^{6}$ EVNs can be called central neurocytoma-like tumors. They are more unusual tumors with biologic behavior and histopathologic characteristics similar to the neoplasms that occur in the lateral ventricles. ${ }^{1-3,7}$ EVNs seemed to exhibit a somewhat larger morphologic spectrum compared with intraventricular neurocytomas. The fourth edition of the WHO classification of tumors of the central nervous system, published in 2007, listed EVN as a new entity. ${ }^{1}$ Case reports have documented involvement of the cerebral hemispheres (commonly the frontal lobe followed by the parietal lobe), thalamus, cerebellum, pons, amygdala, pineal gland, and spinal cord. ${ }^{2-6,8,9}$ EVNs generally manifest through mass effect in the form of seizures and hemiparesis. Few imaging findings have been reported, mostly in case reports. The largest series of neurocytic neoplasms arising within the brain parenchyma is of 35 cases combined from Johns Hopkins Hospital, the Mayo Clinic, and Emory University Hospital. ${ }^{2}$ EVNs typically affect children and young adults, but ages have ranged from 5 to 76 years (median age, 34 years), and no sex predilection has been noted. Ganglion cell differentiation was seen in $66 \%$. Focal GFAP reactivity was present in $46 \%$ despite the lack of apparent astrocytes in H\&E-stained sections.

From a radiographic standpoint, EVNs are generally circumscribed, sometimes large, complex, and variably enhanc- ing masses. The solid portion may have a variable appearance on CT and MR imaging, depending on the cellularity and degree of calcification. They are often partly or mainly cystic, frequently calcified $(>10 \%)$, and may or may not be associated with peritumoral edema. Hemorrhage has been reported sporadically. ${ }^{10}$

The MR imaging findings of this entity have been described in previous case reports and in small series. ${ }^{3-5,7,10-18}$ In many instances, the neurocytoma was well-defined, cystic and heterogeneously solid, and involved the deep white matter or cortical gray matter of the cerebral hemispheres. The solid portion showed varying degrees of enhancement and calcification. EVNs with ganglioid differentiation tended to have more of a cystic component and heterogeneous enhancement appearance. Funato et $\mathrm{al}^{12}$ reported the case of an 11-year-old girl with a neurocytoma. MR imaging demonstrated a large cyst with an enhancing mural nodule arising in the frontal lobe. Histopathologic examination demonstrated ganglioid differentiation. The authors noted no recurrence after subtotal tumor resection. The report by Chan et $\mathrm{al}^{13}$ describes another heterogeneous, T2-hyperintense mass with irregular gadolinium enhancement that mimicked a malignant tumor but was pathologically proved EVN.

In our series, the 2 cases of large, complex cystic and solid masses in the frontal and parietal lobes had many similarities to the previously reported imaging findings of EVNs. ${ }^{2-3} \mathrm{As}$ in the previous examples, they demonstrated cystic change, necrosis, calcification, and hemorrhage. However, an accurate 

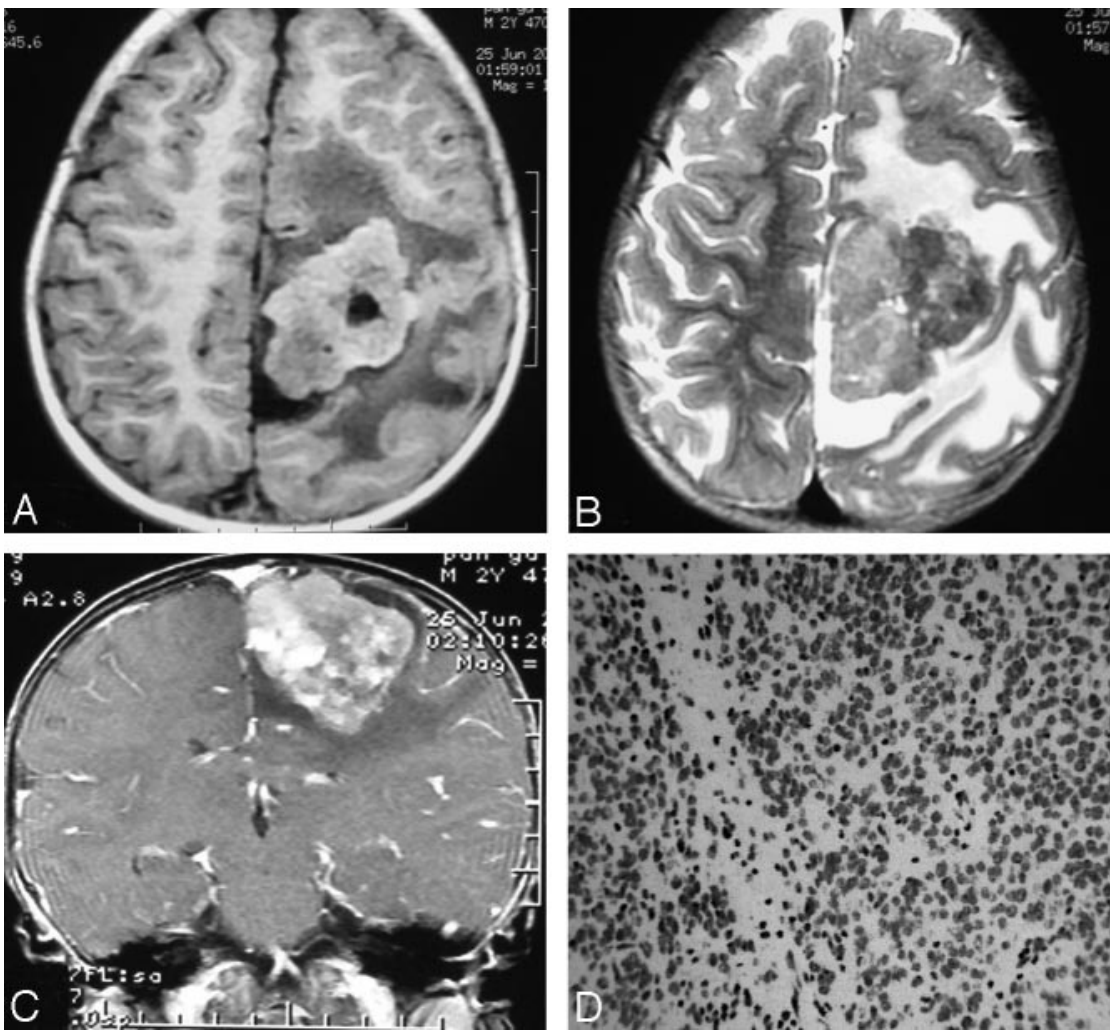

Fig 2. Frontoparietal neurocytoma in a 2-year-old child. $A$, T1-weighted image. $B$, T2-weighted image showing a heterogeneous mass in the frontal and parietal lobes. Low signal intensity in the mass was noted on T2-weighted image. C, Contrast-enhanced T1-weighted image showing extensive heterogeneous enhancement in the solid part of the mass. $D$, H\&E staining showing that the mass was composed of uniform small, round cells.

prospective diagnosis of EVN was difficult because of the rare incidence of this entity. EVNs should be included in the differential diagnosis when a large parenchymal mass with cystic necrosis, calcification, hemorrhage, and extensive enhancement is encountered in a younger patient. EVN may involve the cerebral cortex, closely resembling oligodendroglioma, pilocytic astrocytoma, or ganglioglioma. ${ }^{2}$

Most oligodendrogliomas arise in the white matter. Some have a marked tendency to extend toward and throughout the cerebral cortex. This infiltrative appearance would favor oligodendroglioma compared with EVN. However, typical imaging features of oligodendroglioma include sharply defined margins as well as T1-hypointensity and T2-hyperintensity compared with gray matter on MR imaging and subtle, illdefined enhancement in $15 \%$ to $20 \%$ of cases (associated with higher-grade tumors) ${ }^{19,20}$ Many oligodendrogliomas do not incite the severe perilesional edema seen in cases 1 and 2; in the presence of this type of edema, EVN may be considered. Oligodendroglioma can erode the inner table of the calvaria, which may be a distinguishing feature. Calcification has been seen in $20 \%$ to $91 \%$ of oligodendrogliomas, which is probably less common in EVN. The calcifications of oligodendrogliomas are usually coarse in morphologic appearance and are larger than the "dotted" calcifications encountered in EVN. Calcification can be seen anywhere within an oligodendroglioma; it is especially common where an infiltrating tumor involves the adjacent cortical gray matter, forming an irregular "gyriform" ribbon. In fact, the patient in case 2 was misdiagnosed as having oligodendroglioma because of marked calcification within the neoplasm.
Oligodendroglioma perhaps presents the most challenging diagnosis to consider, even in histopathologic differentiation. Because of the observation that oligodendrogliomas can possess neurocytic differentiation, Perry et $\mathrm{al}^{21}$ suggested a potential, previously unrecognized histogenetic link between oligodendroglioma and extraventricular neurocytoma. Those authors found the characteristic oligodendroglioma-associated chromosome $1 \mathrm{p}$ and $19 \mathrm{q}$ codeletion pattern in $2(17 \%)$ of $12 \mathrm{EVNs}$. Immunohistochemical examination can be helpful in the differentiation of oligodendrogliomas and EVNs. Oligodendrogliomas can show some Syn immunoreactivity but less than that of EVNs, which are strongly reactive for Syn, both in the neuropil and in the perinuclear cytoplasm. ${ }^{2}$ The diagnosis of EVNs can be aided by imaging features that demonstrate a well-demarcated rather than a diffusely infiltrating mass. Although central neurocytomas show similar morphologic features as oligodendrogliomas, EVNs more often display astrocytic, typically pilocytic features and/or ganglionic differentiation on histologic examination.

The differential diagnosis also included several other entities:

High-grade astrocytoma. This neoplasm often occurs in patients aged 40 to 50 years. A complex mass with isointensity or hypointensity on T1-weighted images, and isointensity or hyperintensity on T2-weighted images may be seen. The patient in case 1 was misdiagnosed preoperatively as having a high-grade astrocytoma. However, calcification within astrocytomas is less common than in EVNs. ${ }^{22}$

Primitive neuroectodermal tumor (PNET). These tumors often occur before age 5 years, with the frontal and parietal 

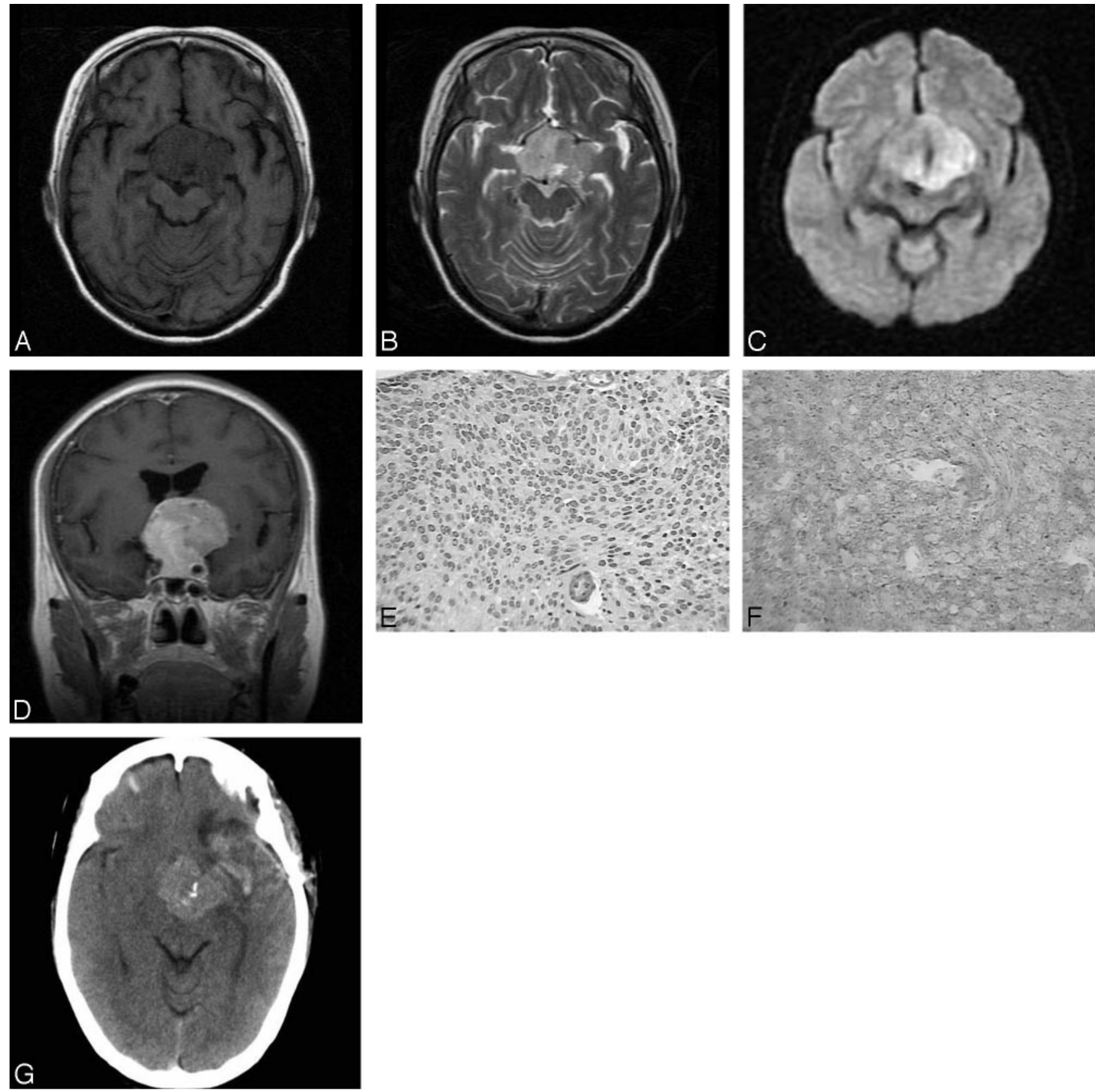

Fig 3. Neurocytoma in the sellar region in a 46-year-old woman. $A$, Axial T1-weighted image. $B$, T2-weighted image showing a sellar and suprasellar mass with $\mathrm{T} 1$ isointensity to gray matter and variable T2 signal intensity in the sellar region. $C$, Diffusion-weighted image showing high signal intensity in the mass. D, Coronal T1-weighted image after contrast administration demonstrated pronounced enhancement within the mass and encroachment on the adjacent vascular structures. $E$, H\&E staining showed that the mass was composed of uniform small, round cells. F, Diffuse positive Syn was demonstrated. G, Postoperative CT image showed a residual suprasellar isoattenuating mass measuring $3.2 \mathrm{~cm} \times 3.0 \mathrm{~cm}$. Calcification is present within the tumor.

lobes being the preferred sites. The PNET may be huge and may be adjacent to lateral ventricles. The solid portion of a PNET may have a low- or high-attenuation appearance, with cystic degeneration, hemorrhage, and calcification. Calcification can be discrete or masslike. PNETs may display complex MR signal intensity and heterogeneous enhancement. Case 2 should be differentiated from this entity.

Ependymoma. These tumors can be classified into 2 types: partly cystic or completely solid. The cystic form often occurs in young adults, whereas the completely solid form often occurs in middle age. The parietal lobe is the preferred site for the partly cystic type. These are often large, with most tumors being larger than $4 \mathrm{~cm}$. Pronounced cystic degeneration and a small solid component at the cerebral cortex may be seen. Calcification is often present and may be linear, dotted, or irregular. Mild or no peritumoral edema may be identified. ${ }^{22}$

Gangliocytoma and ganglioglioma. These tumors often occur in children and in young adults, especially in adults younger than 30 years. The temporal lobe is the predominant site; they can also arise in the cerebellum, brain stem, suprasellar region, and spinal cord. Typical imaging findings are a large cystic mass with a calcified mural nodule. Although the cases of our patients did not fit this pattern, the patient reported by Funato et al, ${ }^{12}$ described before, had a neurocytoma 
with an appearance and presentation similar to those of a ganglioglioma.

Meningioma. The patient in case 3 was misdiagnosed as having a sellar meningioma preoperatively. There are $20 \%$ of meningiomas that can occur in the sellar region, accounting for $10 \%$ of sellar tumors. Nonenhanced coronal and sagittal MR images showed a round, nodular mass above the normal pituitary gland with isointensity on T1-weighted images and high signal intensity on T2-weighted images. Pronounced contrast enhancement was present. A dural tail sign and encroachment on adjacent structures can be present in meningiomas. However, the tumor in case 3 demonstrated a more invasive growth pattern, encompassing the adjacent vascular structures and compressing the optic nerve, resulting in visual loss. Kowalski et $\mathrm{al}^{7}$ did report a skull base neurocytoma showing an invasive pattern. It is noteworthy that their report also indicated that EVNs had variable imaging patterns in different anatomic locations.

Proton MR spectroscopic studies of central neurocytomas and EVNs have recently been published. ${ }^{17,23-25}$ Typical patterns include elevated choline (Cho), decreased creatine $(\mathrm{Cr})$, and decreased $\mathrm{N}$-acetylaspartate (NAA). MR spectroscopy of central neurocytomas shows a 3.35-ppm peak that is not present in other tumors. ${ }^{13}$ This unidentified peak could be because of inositol or glycine and should help to identify a unique marker for central neurocytomas. In the first report on spectroscopic findings of EVN in 2002, ${ }^{17} \mathrm{NAA}$ was shown to be obviously decreased and Cho elevated. Ueda et $\mathrm{al}^{18}$ demonstrated that a combination of prominent Cho resonance and detectable Cr resonance was a common feature of both intraventricular and extraventricular neurocytomas. These findings will be helpful in the differentiation of EVNs from other tumors in the brain parenchyma.

\section{Conclusions}

EVN is a rare neoplasm that can have significant overlap in imaging appearance with other primary brain neoplasms; therefore, it is difficult to make an accurate preoperative diagnosis. EVN should be considered in the differential diagnosis when a large cerebral parenchymal mass with cystic necrosis, calcification, and/or hemorrhage foci, and extensive enhancement is encountered in younger patients.

\section{References}

1. Louis DN, Ohgaki H, Wiestler OD, et al. The 2007 WHO classification of tumours of the central nervous system. Acta Neuropathol 2007;114:97-109
2. Brat DJ, Scheithauer BW, Eberhart CG, et al. Extraventricular neurocytomas: pathologic features and clinical outcome. Am J Surg Pathol 2001; 25:1252-60

3. Giangaspero F, Cenacchi G, Losi L, et al. Extraventricular neoplasms with neurocytoma features. A clinicopathological study of 11 cases. Am J Surg Pathol 1997;21:206-12

4. Brown DM, Karlovits S, Lee LH, et al. Management of neurocytomas: case report and review of the literature. Am J Clin Oncol 2001;24:272-78

5. Tortori-Donati P, Fondelli MP, Rossi A, et al. Extraventricular neurocytoma with ganglionic differentiation associated with complex partial seizures. AJNR Am J Neuroradiol 1999;20:724-27

6. Sharma MC, Deb P, Sharma S, et al. Neurocytoma: a comprehensive review. Neurosurg Rev 2006;29:270-85

7. Kowalski RJ, Prayson RA, Lee JH. Skull base neurocytoma: case report and review of the literature of extraventricular neurocytomas. Skull Base 2002;12:59-65

8. Schmidt MH, Gottfried ON, von Koch CS, et al. Central neurocytoma: a review. J Neurooncol 2004;66:377-84

9. Lee J, Chang SM, McDermott MW, et al. Intraventricular neurocytomas. Neurosurg Clin N Am 2003;14:483-508

10. Ritz R, Roser F, Bornemann A, et al. Extraventricular neurocytoma presenting with intratumoral hemorrhage. Clin Neuropathol 2005;24:101-05

11. Sgouros S, Carey M, Aluwihare N, et al. Central neurocytoma: a correlative clinicopathologic and radiologic analysis. Surg Neurol 1998;49:197-204

12. Funato $\mathrm{H}$, Inoshita $\mathrm{N}$, Okeda $\mathrm{R}$, et al. Cystic ganglioneurocytoma outside the ventricular region. Acta Neuropathol (Berl) 1997;94:95-98

13. Chan A, McAbee G, Queenan J, et al. Ganglioneurocytoma mimicking a malignant tumor: Case report with a literature review of the MRI appearance of neurocytomas and gangliogliomas. J Neuroimaging 2001;11:47-50

14. Shin JH, Lee HK, Lee JK, et al. MR imaging and histopathologic findings of a case of cerebral ganglioneurocytoma. Korean J Radiol 2002;3:214-17

15. Mrak RE, Yasargil MG, Mohapatra G, et al. Atypical extraventricular neurocytoma with oligodendroglioma-like spread and an unusual pattern of chromosome $1 p$ and 19q loss. Hum Pathol 2004;35:1156-59

16. Park KJ, Kim JH, Park YK, et al. Extraventricular cystic neurocytoma. J Korean Neurosurg Soc 2004;35:102-05

17. Möller-Hartmann W, Krings T, Brunn A, et al. Proton magnetic resonance spectroscopy of neurocytoma outside the ventricular region-case report and review of the literature. Neuroradiology 2002;44:230-34

18. Ueda F, Suzuki M, Matsui O, et al. Automated MR spectroscopy of intra- and extraventricular neurocytomas. Magn Reson Med Sci 2007;6:75-81

19. van den Bent MJ. Diagnosis and management of oligodendroglioma. Semin Oncol 2004;31:645-52

20. Olson JD, Riedel E, DeAngelis LM. Long-term outcome of low-grade oligodendroglioma and mixed glioma. Neurology 2000;54:1442-48

21. Perry A, Scheithauer BW, Macaulay RJ, et al. Oligodendrogliomas with neurocytic differentiation. A report of 4 cases with diagnostic and histogenetic implications. J Neuropathol Exp Neurol 2002;61:947-55

22. Zhang B, Luo B, Zhang Z, et al. Central neurocytoma: a clinicopathological and neuroradiological study. Neuroradiology 2004;46:888-95

23. Jayasundar $\mathrm{R}$, Shah $\mathrm{T}$, Vaishya $\mathrm{S}$, et al. In vivo and in vitro $M R$ spectroscopic profile of central neurocytomas. J Magn Reson Imaging 2003;17:256-60

24. Krishnamoorthy T, Radhakrishnan VV, Thomas B, et al. Alanine peak in central neurocytomas on proton MR spectroscopy. Neuroradiology 2007;49: 551-54

25. Yeh IB, Xu M, Ng WH, et al. Central neurocytoma: typical magnetic resonance spectroscopy findings and atypical ventricular dissemination. Magn Reson Imaging 2008;26:59-64 